\title{
A Comparison of AKIN, KDIGO, and RIFLE Definitions to Diagnose Acute Kidney Injury and Predict the Outcomes after Cardiac Surgery in a South Asian Cohort
}

\author{
Sonia Yaqub ${ }^{a}$ Shiraz Hashmi ${ }^{\text {b }}$ Muhammad Kashif Kazmi ${ }^{a} \quad$ Arzina Aziz Ali ${ }^{a}$ \\ Tasneem Dawood ${ }^{a}$ Hasanat Sharif ${ }^{b}$ \\ aSection of Nephrology, Department of Medicine, Aga Khan University Hospital, Karachi, Pakistan; ${ }^{\text {bSection of }}$ \\ Cardiothoracic Surgery, Department of Surgery, Aga Khan University Hospital, Karachi, Pakistan
}

\section{Keywords}

Acute kidney injury · Cardiac surgery · Acute Kidney Injury Network · Kidney Disease: Improving Global Outcomes ·

Risk, injury, failure, loss, and end-stage kidney disease

\begin{abstract}
Background: Acute kidney injury (AKI) after cardiac surgery is associated with an increased mortality and morbidity. Different definitions for AKI have been used such as Acute Kidney Injury Network (AKIN), Kidney Disease: Improving Global Outcomes (KDIGO), or risk, injury, failure, loss, end-stage kidney disease (RIFLE). Each of these definitions has their own benefits and limitations for predicting the degree of AKI and adverse outcomes following cardiac surgery. This study was aimed to compare the three AKI definitions to diagnose AKI and their predictive ability for mortality and morbidity after isolated coronary artery bypass surgery (CABG) in a South Asian cohort. Material and Methods: A single-center retrospective review was conducted on 1,508 patients having undergone isolated CABG surgery from January 2015 to January 2019. AKI was assessed on three definitions, and comparative receiver operating characteristics curves were built
\end{abstract}

Karger@karger.com www.karger.com/crm

Karger $\stackrel{\text { ' }}{5}$

GOPEN ACCESS
(C) 2022 The Author(s)

Published by S. Karger AG, Basel

This is an Open Access article licensed under the Creative Commons Attribution-NonCommercial-4.0 International License (CC BY-NC) (http://www.karger.com/Services/OpenAccessLicense), applicable to the online version of the article only. Usage and distribution for commercial purposes requires written permission. against the outcomes to assess discriminative power of each. Results: Mean age of participants was $59.43( \pm 1.12)$ years, predominantly males (82.6\%). Patients with AKI were elder, more likely to be diabetic and hypertensive. AKI by any definition occurred in $58.7 \%(885 / 1,508)$ patients. Frequency of AKI was 508 (33.7\%), 517 (34.4\%), and 871 (57.8\%) on AKIN, KDIGO, and RIFLE criteria, respectively. The proportion of patients with RIFLE-risk 619 (41\%) was greater compared with AKIN stage 1 (342 [22.7\%]) and KDIGO stage 1 (330 [21.9\%]), while for stages 2 and 3 the proportions were comparable across the three systems. Area under the curve (AUC) for 30day mortality for AKIN was $(0.786$, [95\% Cl: $0.764-0.806])$, KDIGO: (0.796, [95\% Cl: 0.775-0.816]) and for RIFLE (0.844, [95\% Cl: 0.825-0.862]). AUC for overall morbidity was in undesirable ranges (i.e., $>0.5-\leq 0.7$ ) for all three definitions. Conclusion: In a South Asian cohort, performance of AKIN and KDIGO criteria was comparable to diagnose AKI, while RIFLE definition, though overestimated the incidence of AKI particularly stage 1 , and had an excellent discriminatory power to predict mortality compared to other two definitions.

(C) 2022 The Author(s)

Published by S. Karger AG, Basel
Correspondence to:

Sonia Yaqub, sonia.yaqub@aku.edu 


\section{Introduction}

Acute kidney injury (AKI) following a coronary artery bypass surgery (CABG) is associated with several adverse outcomes including increase in the long-term risk of endstage kidney disease (ESKD), length of hospital stay, healthcare costs, increase in morbidity, and short- as well as long-term mortality $[1,2]$. Depending upon the definitions used the incidence of AKI after cardiac surgery varies from $5 \%$ to $42 \%$ in various settings and regions [3]. Risk, injury, failure, loss, and ESKD (RIFLE), Acute Kidney Injury Network (AKIN), and Kidney Disease: Improving Global Outcomes (KDIGO) are the three widely accepted consensus definitions for the diagnosis of AKI in noncardiac surgery settings. However, currently, there is no standardized or universally accepted criterion or definition of AKI in post-cardiac surgery patients. Having a standard criterion for diagnosing and classifying AKI would enhance the ability to improve the management and outcomes of these patients. There is scarce data on comparison of these definitions in their ability to diagnose AKI and to predict outcomes in the South Asian population. This comparison is of utmost importance in accurately predicting the degree of AKI and planning the appropriate management strategies keeping in view the benefit and limitation of each criterion. Our study aimed to compare these three AKI definitions to determine frequency of AKI and their predictive ability for all-cause mortality and morbidity after isolated CABG surgery.

\section{Materials and Methods}

\section{Study Population}

We performed a retrospective review of prospectively collected data at a single tertiary care center in Southern Pakistan. Demographic and clinical data were analyzed to determine the occurrence of AKI using three definitions in patients, who had undergone isolated CABG surgery from January 2015 to January 2019. The study was approved by Institutional Research Ethics Committee and complies with the Declaration of Helsinki research study protocol.

\section{Inclusion and Exclusion Criteria}

Patients aged 18 years or older who had undergone isolated CABG during the designated study period were included in this study. Patients were excluded if they were undergoing emergent surgery, had a history of exposure to contrast medium in the 3 days preceding surgery or within the first $24 \mathrm{~h}$ post-surgery, or any missing pre- or postoperative measurement of serum creatinine (SCr), diagnosed with cancer, any systemic infection during their hospital stay, had a kidney transplant or were on maintenance hemodialysis or peritoneal dialysis. Patients with known preexisting kidney impairment defined as a preoperative estimated glomerular filtration rate (eGFR) of less than $60 \mathrm{~mL} / \mathrm{min} / 1.73 \mathrm{~m}^{2}$ according to the Chronic
Kidney Disease Epidemiology Collaboration equation (validated for Pakistani population) were also excluded from this study [4].

\section{Operational Definitions}

Acute Kidney Injury

The incidence and stages of AKI were determined using the three definitions from AKIN, KDIGO, and RIFLE (Table 1). Baseline SCr was measured on the day of admission-day- 0 , and highest postoperative creatinine was used to assess the change in the values. Patients were classified based on absolute changes in SCr according to AKIN and KDIGO classifications and for RIFLE, eGFR was used instead of SCr criterion. eGFR was calculated using modified Chronic Kidney Disease Epidemiology Collaboration equation validated for Pakistani population [4]. RIFLE subdivides AKI into three severity stages (risk, injury, and failure and loss and ESKD), whereas both AKIN and KDIGO use only three severity stages (stages 1,2, and 3). Operationally, stage 1 corresponds to risk, stage 2 corresponds to injury, and stage 3 corresponds to failure. For the sake of clarity and simplicity, we used stages 1,2, and 3 across all definitions. Patients with stages L and $\mathrm{E}$ of the RIFLE classification were not included in the analysis. The urine output criteria for either definition system were not used because our database did not contain six or 12-h urine output data for all patients.

Operative Mortality [5]: All-cause deaths occurr during the hospitalization in which the CABG was performed and those deaths occur after discharge from the hospital but within 30 days of procedure.

Morbidities [5]: Overall morbidities were defined as occurrence of one or more than one of the following:

- Prolonged ventilation: Prolonged postoperative ventilation $>24$ $\mathrm{h}$ (includes hours from OR exit until extubation, plus any additional hours following reintubation including reintubation).

- Reopening: Patients reopened for bleeding, tamponade, graft occlusion, or other cardiac reasons within 30 days of index surgery.

- Stroke: Any confirmed neurological deficit caused by disturbance in cerebral blood supply that does not resolve within 24 $\mathrm{h}$ within 30 days of the index surgery.

- Deep sternal wound infection: Infections involving muscle, bone, and of mediastinum requiring operative intervention within 30 days postoperatively.

Must have all of the following conditions:

- Wound opened with excision of tissue incision and drainage or reexploration of mediastinum.

- Positive culture unless patient on antibiotics at time of culture or no culture obtained.

- Treatment with antibiotics beyond perioperative prophylaxis.

\section{Statistical Analysis}

Data analysis was performed on SPSS version 22 and MedCalc 19.5.3 analytical software. Descriptive characteristics of both groups were compared using independent $t$ test for continuous variables and $\chi^{2}$ or Fisher exact test for categorical variables. Absolute percent change was computed using baseline SCr or eGFR and highest postoperative SCr during the course of hospitalization and categorized as per definitions shown in Table 1. Comparative receiver operating characteristic curves were built and sensitivity, specificity, and area under the curves (AUCs) with 95\% confidence interval were computed to assess the performance of all three AKI definitions for predicting the outcomes. A $p$ value of $<0.05$ was considered significant.
Cardiorenal Med 2022;12:29-37 DOI: $10.1159 / 000523828$
Yaqub/Hashmi/Kazmi/Aziz Ali/Dawood/ Sharif 
Table 1. RIFLE, AKIN, and KDIGO definitions for staging of AKI

\begin{tabular}{|c|c|c|c|}
\hline \multicolumn{4}{|l|}{ RIFLE } \\
\hline Risk & & $\begin{array}{l}\text { SCr increase to } 1.5 \text {-fold or GFR decrease }>25 \% \\
\text { from baseline }\end{array}$ & $<0.5 \mathrm{~mL} / \mathrm{kg} / \mathrm{h}$ for $6 \mathrm{~h}$ \\
\hline Injury & & $\begin{array}{l}\mathrm{SCr} \text { increase to } 2.0 \text {-fold or GFR decrease }>50 \% \\
\text { from baseline }\end{array}$ & $<0.5 \mathrm{~mL} / \mathrm{kg} / \mathrm{h}$ for $12 \mathrm{~h}$ \\
\hline Failure & & $\begin{array}{l}\text { SCr increase to } 3.0 \text {-fold or GFR decrease }>75 \% \\
\text { from baseline or } \mathrm{SCr} \geq 4 \mathrm{mg} / \mathrm{dL} \text { with an acute } \\
\text { increase of at least } 0.5 \mathrm{mg} / \mathrm{dL}\end{array}$ & Anuria for $12 \mathrm{~h}$ \\
\hline \multicolumn{4}{|l|}{ AKIN } \\
\hline 1 & $\begin{array}{l}\mathrm{SCr} \text { increase } \geq 0.3 \mathrm{mg} / \mathrm{dL}(\geq 26.5 \mu \mathrm{mol} / \mathrm{L} \text { ) or increase to } 1.5-2.0- \\
\text { fold from baseline }\end{array}$ & & $<0.5 \mathrm{~mL} / \mathrm{kg} / \mathrm{h}$ for $6 \mathrm{~h}$ \\
\hline 2 & SCr increase $>2.0-3.0$-fold from baseline & & $<0.5 \mathrm{~mL} / \mathrm{kg} / \mathrm{h}$ for $12 \mathrm{~h}$ \\
\hline 3 & $\begin{array}{l}\text { SCr increase }>3.0 \text {-fold from baseline or } \mathrm{SCr} \geq 4.0 \mathrm{mg} / \mathrm{dL}(\geq 354 \\
\mu \mathrm{mol} / \mathrm{L}) \text { with an acute increase of at least } 0.5 \mathrm{mg} / \mathrm{dL}(44 \mu \mathrm{mol} / \mathrm{L}) \\
\text { or need for RRT }\end{array}$ & & $\begin{array}{l}<0.3 \mathrm{~mL} / \mathrm{kg} / \mathrm{h} \text { for } 24 \mathrm{~h} \\
\text { or anuria for } 12 \mathrm{~h} \text { or } \\
\text { need for RRT }\end{array}$ \\
\hline 2 & SCr 2.0-2.9 times baseline & & $\begin{array}{l}<0.5 \mathrm{~mL} / \mathrm{kg} / \mathrm{h} \text { for } \\
\geq 12 \mathrm{~h}\end{array}$ \\
\hline 3 & $\begin{array}{l}\mathrm{SCr} 3.0 \text { times baseline or increase in } \mathrm{SCr} \text { to } \geq 4.0 \mathrm{mg} / \mathrm{dL} \text { or } \\
\text { initiation of RRT }\end{array}$ & & $\begin{array}{l}<0.3 \mathrm{~mL} / \mathrm{kg} / \mathrm{h} \text { for } \geq 24 \\
\mathrm{~h} \text { or anuria for } \geq 12 \mathrm{~h}\end{array}$ \\
\hline
\end{tabular}

GFR, glomerular filtration rate; RRT, renal replacement therapy; AKI, acute kidney injury.

Table 2. Comparison of baseline clinical characteristics of patients with AKI and no AKI with any of the three definitions, $n=$ 1,508

\begin{tabular}{lllr}
\hline Variable & $\begin{array}{l}\text { AKI } \\
(n=885)(58.7 \%)\end{array}$ & $\begin{array}{l}\text { No AKI } \\
(n=623)(41.3 \%)\end{array}$ & $p$ value* \\
\hline Age, years & $60.5 \pm 9.6$ & $57.9 \pm 9.3$ & $<0.001$ \\
Gender (male) & $720(81.4)$ & $526(84.4)$ & 0.129 \\
Body mass index, kg/m ${ }^{2}$ & $27.3 \pm 4.6$ & $27.1 \pm 4.8$ & 0.384 \\
Diabetes mellitus & $494(55.8)$ & $312(50.1)$ & 0.028 \\
Hypertension & $690(78.1)$ & $436(70.1)$ & $<0.001$ \\
Preoperative SCr, mg/dL & $1.0 \pm 0.3$ & $0.9 \pm 0.2$ & 0.068 \\
Postoperative SCr, mg/dL & $1.5 \pm 0.6$ & $1.1 \pm 0.3$ & $<0.001$ \\
Preoperative eGFR & $76.2 \pm 17.6$ & $75.0 \pm 16.0$ & 0.194 \\
Postoperative eGFR & $46.2 \pm 18.3$ & $68.0 \pm 16.0$ & $<0.001$ \\
Left ventricular ejection fraction, \% & $49.8 \pm 12.5$ & $50 \pm 11.5$ & 0.815 \\
\hline \multicolumn{2}{c}{ Mean \pm standard deviation. eGFR, estimated glomerular filtration rate; CKD-EPI, Chronic } \\
Kidney Disease Epidemiology Collaboration. ${ }^{*}$ Student's $t$ test or $x^{2} /$ Fisher exact test. ${ }^{\dagger}$ eGFR \\
mL/min/1.73 m $^{2}$ calculated on CKD-EPI equation for South Asians.
\end{tabular}

\section{Results}

A total 1,508 cases have undergone isolated CABG surgery were recruited. The mean age of the study population was $59.43 \pm 1.12$ years with predominantly males (82.6\%). Table 2 shows baseline characteristics of the study population. Patients who were elderly $(p<0.001)$ and those with comorbid conditions of diabetes $(p=$
$0.028)$ and hypertension $(p<0.001)$ were more likely to develop AKI.

Table 3 describes perioperative characteristics of the study population. Development of AKI was associated with longer perfusion and cross-clamp times, use of intraoperative blood products, longer hospital stay, prolonged ventilation, and deep sternal wound infection ( $p$ $<0.05$ for all). Of those who developed AKI, only 10 pa- 
Table 3. Perioperative characteristics of study population with $\mathrm{AKI}$ and without $\mathrm{AKI}$, $n=1,508$

\begin{tabular}{|c|c|c|c|}
\hline Variable & $\begin{array}{l}\text { AKI } \\
(n=885)(58.7 \%)\end{array}$ & $\begin{array}{l}\text { No AKI } \\
(n=623)(41.3 \%)\end{array}$ & $p$ value* \\
\hline Over all morbidity ${ }^{\ddagger}$ & $94(10.6)$ & $32(5.1)$ & $<0.001$ \\
\hline Perfusion time, min & $109.6 \pm 62.2$ & $101.3 \pm 45.1$ & 0.004 \\
\hline Cross clamp time, min & $68.7 \pm 25.6$ & $64.0 \pm 23.1$ & $<0.001$ \\
\hline Intraoperative use of blood products & $461(52.2)$ & $274(44.1)$ & 0.002 \\
\hline Prolonged ventilation & $63(7.1)$ & $19(3.0)$ & $<0.001$ \\
\hline Reopen for any reason & $29(3.3)$ & $12(1.9)$ & 0.112 \\
\hline Stroke & $10(1.1)$ & $2(0.3)$ & 0.138 \\
\hline Deep sternal wound infection & $16(1.8)$ & $2(0.3)$ & 0.009 \\
\hline Length of stay, days & $8.7 \pm 5.4$ & $7.8 \pm 3.8$ & 0.004 \\
\hline Operative mortality ${ }^{\dagger}$ & $34(3.8)$ & $1(0.2)$ & $<0.001$ \\
\hline
\end{tabular}

Mean \pm standard deviation. * Student $t$ test or $x^{2} /$ Fisher exact test. ${ }^{\ddagger}$ Overall morbidity is as defined occurrence of any one or more adverse events during 30-day postoperative period after index procedure that includes prolonged ventilation of $>24 \mathrm{~h}$; reopened for bleeding, tamponade, graft occlusion; any neurological deficit including stroke that does not resolve within $24 \mathrm{~h}$ of index procedure, and deep sternal wound infection. ${ }^{\dagger}$ Thirty-day all-cause mortality.

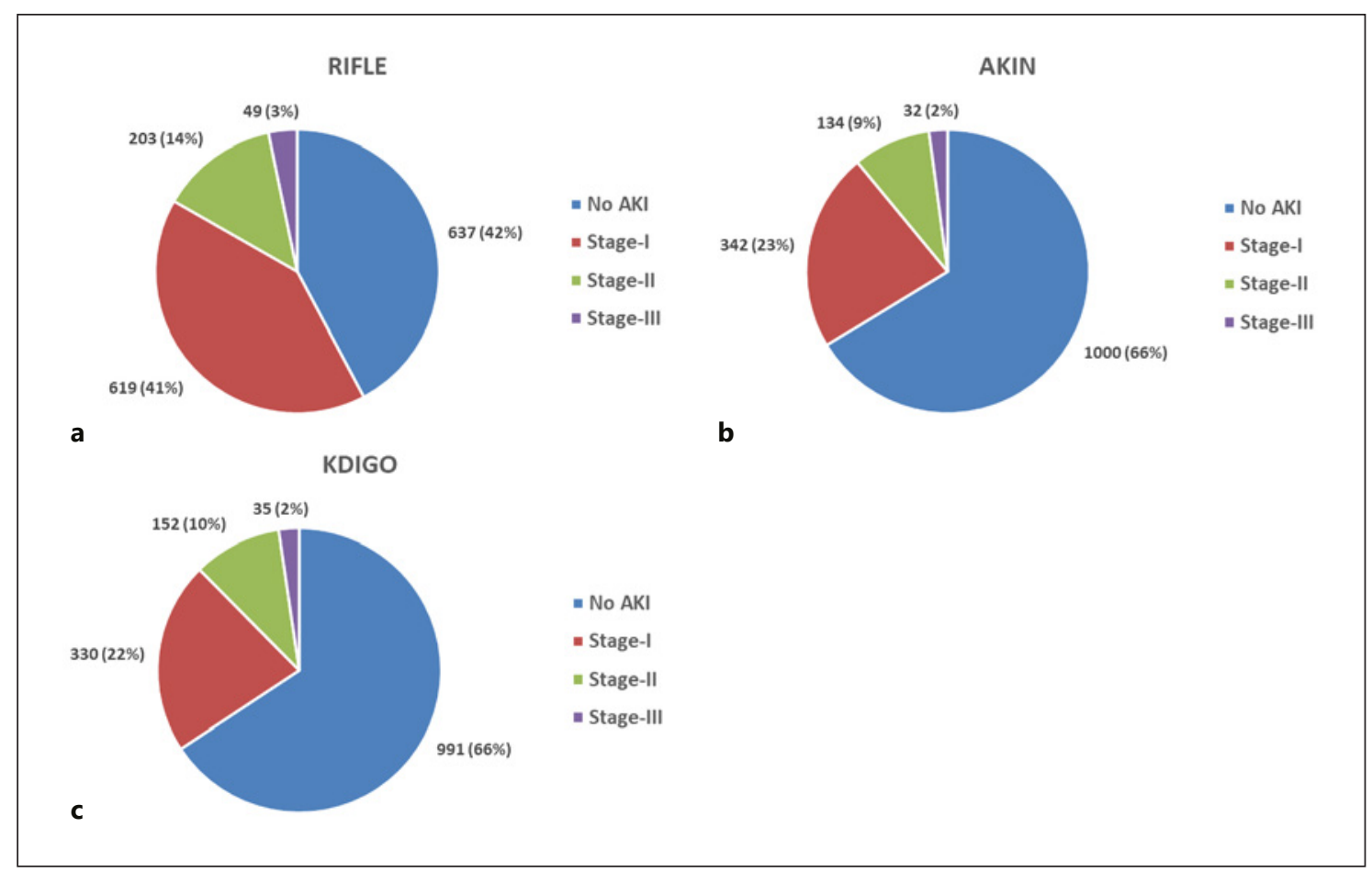

Fig. 1. Incidence and stages of AKI according to RIFLE (a), AKIN (b), and KDIGO (c) definitions.

tients (1.1\%) required kidney replacement therapy (KRT).

\section{Incidence of AKI Using Different Definitions}

AKI by any definition was estimated in $58.7 \%$ $(885 / 1,508)$ of cardiac surgery patients. Using the dif- ferent criteria, the incidence of AKI for AKIN was 508 (33.7\%), KDIGO 517 (34.3\%) and for RIFLE, it was $871(57.8 \%)$. The incidence of different stages of AKI according to the three definitions is shown in Figure $1 \mathrm{a}-\mathrm{c}$. 
Table 4. Sensitivity, specificity, and AUC for 30 day operative mortality by the three AKI definitions

\begin{tabular}{llll}
\hline $\begin{array}{l}\text { AKI definition and } \\
\text { operative mortality }\end{array}$ & AUC $(95 \% \mathrm{Cl})$ & $\begin{array}{l}\text { Sensitivity, Specificity, } \\
\%\end{array}$ \\
\hline AKIN & $0.786(0.764-0.806)$ & 62.16 & 85.18 \\
KDIGO & $0.796(0.775-0.816)$ & 64.86 & 84.36 \\
RIFLE & $0.844(0.825-0.862)$ & 73.0 & 88.00 \\
\hline
\end{tabular}

AKIN, Acute Kidney Injury Network; KDIGO, Kidney Disease: Improving Global Outcomes; RIFLE, Risk, Injury and Failure; AUC, Area Under the Curve; $\mathrm{Cl}$, Confidence Interval. ${ }^{\dagger}$ All-cause 30-day mortality.
Table 5. Difference of AUC for 30-day operative mortality between the three AKI definitions

\begin{tabular}{lll}
\hline Difference AUC & AUC difference $(95 \% \mathrm{CI})$ & $p$ value \\
\hline AKIN and KDIGO & $0.0101(-0.00269$ to 0.0228$)$ & 0.122 \\
AKIN and RIFLE & $0.0585(0.0200-0.0971)$ & 0.003 \\
KDIGO and RIFLE & $0.0485(0.0105-0.0865)$ & 0.012 \\
\hline
\end{tabular}

AKIN, Acute Kidney Injury Network; KDIGO, Kidney Disease: Improving Global Outcomes; RIFLE, Risk, Injury and Failure; AUC, Area Under the Curve; $\mathrm{Cl}$, Confidence Interval.

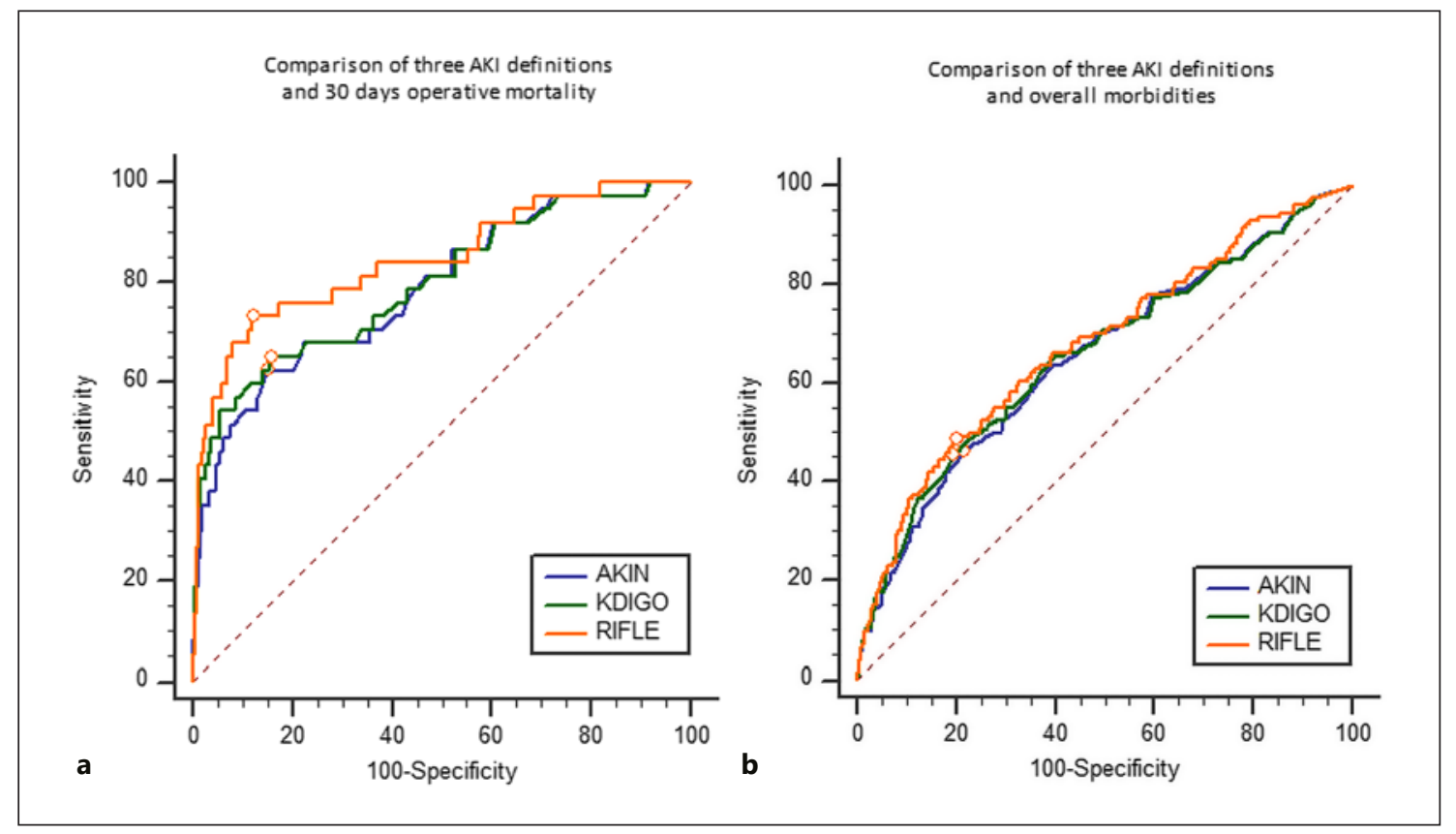

Fig. 2. Comparative ROC curves show association of 30-day mortality (a) and overall morbidity (b) with the three AKI definitions. ROC, receiver operating characteristics.

Thirty-Day Morbidity and Mortality

Overall mortality rate was $35(2.3 \%)$ and was significantly associated with AKI, 34 (3.8\%) compared to those without AKI (1 $[0.2 \%], p$ value $<0.001)$. Receiver operating characteristic curve areas for 30-day mortality for AKIN: (0.786, [95\% CI: 0.764-0.806]), KDIGO: (0.796, [95\% CI: $0.775-0.816])$, and RIFLE (0.844, [95\% CI: $0.825-0.862]$ ). AUC showed a good discrimination ability by RIFLE for operative mortality, whereas AKIN and KDIGO have comparable ability to predict the mortality, the difference in AUC was not significant ( $p$ value: 0.122 ) whereas differences between AKIN and RIFLE and KDIGO and RIFLE were significantly different ( $p$ value were $<0.05$ for both), Tables 4 and 5, Figure 2a. AUC for overall morbidity fell in undesirable ranges $(>5.0-\leq 0.7)$ for all three definitions [6]. Alike mortality, no significant difference was observed between AKIN and KDIGO definitions, however, differences between AKIN and RIFLE, and KDIGO and RIFLE were statistically significant, $p<$ 0.05 (Tables 6, 7; Fig. 2b). 
Table 6. Sensitivity, specificity and AUC for overall morbidities by the three AKI definitions

\begin{tabular}{llll}
\hline $\begin{array}{l}\text { AKI definition and } \\
\text { overall morbidity }\end{array}$ & AUC $(95 \% \mathrm{Cl})$ & $\begin{array}{l}\text { Sensitivity, } \\
\%\end{array}$ & $\begin{array}{l}\text { Specificity, } \\
\%\end{array}$ \\
\hline AKIN & $0.652(0.628-0.676)$ & 46.0 & 78.8 \\
KDIGO & $0.658(0.633-0.682)$ & 45.2 & 81.0 \\
RIFLE & $0.675(0.651-0.699)$ & 48.4 & 80.1 \\
\hline
\end{tabular}

AKIN, Acute Kidney Injury Network; KDIGO, Kidney Disease: Improving Global Outcomes; RIFLE, Risk, Injury and Failure; AUC, Area Under the Curve; $\mathrm{Cl}$, Confidence Interval. ${ }^{\dagger}$ Overall morbidity includes STS.

\section{Discussion}

In this cohort, we found that RIFLE criterion was superior to AKIN and KDIGO in terms of prognostic value to predict mortality after isolated CABG surgery. The current study is the first report from the region to have compared the ability of the three AKI criteria (AKIN, KDIGO, and RIFLE) to determine frequency of AKI and to investigate their prognostic value in post-CABG patients with relatively preserved baseline kidney function (preoperative eGFR $>60 \mathrm{~mL} / \mathrm{min} / 1.73 \mathrm{~m}^{2}$ ). AKI is associated with significant morbidity and mortality, and the identification of risk of post-CABG AKI is of paramount importance because it increases the physician's awareness of the presence of risk for kidney injury and allows timely intervention and management.

The RIFLE criteria were introduced in 2004 with the aim of standardizing the definition of AKI $[7,8]$; however, it was modified shortly after by the AKIN [8-10] when evidence suggested that even small changes in SCr may be associated with increased mortality. The AKIN criteria classified these minor changes in SCr values over a period of $48 \mathrm{~h}$ to determine the change in creatinine. Later in 2012, the AKI study group, "KDIGO" further modified the AKI definition by considering changes in creatinine within $48 \mathrm{~h}$ or a decline in the GFR over 7 days [11]. The KDIGO guidelines define AKI as an increase in SCr concentration $\geq 0.3 \mathrm{mg} / \mathrm{dL}$ within $48 \mathrm{~h}$. The aforementioned definitions of AKI are widely accepted after being independently validated in multiple studies in general population [12]. However, in patients undergoing cardiac surgery, each of definition systems has their own benefits and limitations for predicting the degree of AKI as well as adverse outcomes such as need for KRT, morbidity, and mortality. Hence, the quest to determine the best definition of AKI
Table 7. Difference of AUC for overall morbidities between the three AKI definitions

\begin{tabular}{lll}
\hline Difference AUC & AUC difference $(95 \% \mathrm{Cl})$ & $p$ value \\
\hline AKIN and KDIGO & $0.00537(-0.00315$ to 0.0139$)$ & 0.217 \\
AKIN and RIFLE & $0.0227(0.00909-0.0362)$ & 0.001 \\
KDIGO and RIFLE & $0.0173(0.00644-0.0282)$ & 0.002 \\
\hline
\end{tabular}

AKIN, Acute Kidney Injury Network; KDIGO, Kidney Disease: Improving Global Outcomes; RIFLE, Risk, Injury and Failure; AUC, Area under the Curve; $\mathrm{Cl}$, confidence interval.

after cardiac surgery with the largest impact on the outcome has not yet ended.

Only a few studies have reported frequency of AKI after isolated CABG surgery and the frequency or incidence vary depending upon the definition or criteria used to diagnose AKI. We found that the frequency of AKI by any of the three definitions was $58.7 \%$. This percentage of postoperative $\mathrm{AKI}$ is rather high, as compared to previous reports from rest of the world [13-16]. This difference could be attributable to diversity of patients' characteristics, nature of cardiac surgery procedures, and differences among the types of treatment facilities causing interstudy heterogeneity and thus limiting comparison among studies. A Chinese study reported incidence of AKI as $27.9 \%$ using the KDIGO definition [16]. Amini et al. [13] from Iran assessed the frequency and severity of AKI based on AKIN definition and found it to be $15.8 \%$. Ortega-Loubon et al. [15] from Spain found an even lower incidence of AKI (12.4\%) with RIFLE criteria. A retrospective observational study to evaluate AKI outcomes on 1,260 patients from a multiethnic Southeast Asian population, who underwent a primary isolated CABG operation, found an overall incidence of $36.2 \%$ [14]. They observed a significantly higher risk of developing AKI postCABG in patients of Malay and Indian ethnicity had compared to the Chinese. Another study based upon Southeast Asian population demonstrated a similar relationship [17]. The higher frequency of AKI in our study may corroborate with growing evidence that ethnicity plays a significant role in diabetes, atherosclerosis, coronary heart disease, and its associated complications, including AKI $[18,19]$. Likewise, a meta-analysis comparing data across different continents reported a higher incidence of AKI in Asia (33.5\%), followed by South America and North America (28.6\% and 22.0\%, respectively) [20]. Moreover, we speculate that variations dur-
Cardiorenal Med 2022;12:29-37 DOI: $10.1159 / 000523828$
Yaqub/Hashmi/Kazmi/Aziz Ali/Dawood/ Sharif 
ing conduct of cardiopulmonary bypass such as perfusion practices, monitoring and anesthesia practices, use of vasoactive drugs and regulation of body temperature were not standardized and could have been a likely contributing factor towards a higher burden of postoperative AKI in our study cohort. Larger, multicenter prospective studies ensuring standardized practices with adherence to evidence-based and internationally accepted protocols would help provide a better understanding of determinants of AKI and devise local guidelines to help bring in uniformity in management to ensure patient safety and quality of clinical care for best outcomes.

Application of the three definitions resulted in different AKI incidences in our study. We observed that the largest percentage of patients developed AKI defined by RIFLE criteria (57.8\%) followed by AKIN (33.7\%) and KDIGO (34.3\%). Further, the proportion of patients with RIFLE-risk (41\%) was greater compared with AKIN stage $1(22.7 \%)$ and KDIGO stage $1(21.9 \%)$. The fraction of patients with AKIN stage 2, KDIGO stage 2, and RIFLEinjury was comparable at $8.9 \%, 10.1 \%$, and $13.5 \%$, respectively. The incidence of stage 3 of AKIN, KDIGO, and RIFLE-failure was similar at $2.1 \%, 2.3 \%$, and $3.2 \%$, respectively. We found that RIFLE identified more stage 1 (equivalent to RIFLE-risk) events compared to AKIN and KDIGO. Among pediatric population undergoing cardiac surgery, RIFLE (pediatric) has also been found to be the most sensitive test in detecting AKI as well, especially in infants [21]. The need to recognize stage 1 AKI cannot be overemphasized since early detection and intervention may prevent progression to stage 2 or stage $3 \mathrm{AKI}$ and improve outcomes. We observed that the incidence and staging of AKI according to the KDIGO were comparable to the AKIN classification for the study population. Moreover, there was an overall agreement of the three classifications with increasing severity of AKI (i.e., stages 2 and 3). We used RIFLE criteria based upon changes in eGFR, rather than absolute values of SCr, and hence, it would provide age- and gender-adjusted estimates of incidence of AKI and its various stages. The caveats of relying on SCr are well known especially for the early detection of AKI because rises in SCr are delayed following the kidney insult. Hemodilution from cardiopulmonary bypass can cause initial SCr levels to appear lower than preoperative levels, even if substantial kidney injury occurred during surgery. This is more relevant in setting of normal preoperative kidney function as in our study population since GFR may decrease significantly with only minimal effect on SCr early on in development of AKI. A meta-analysis comparing the three definitions in patients after cardiac surgery found that the incidence of AKI was highest with AKIN [20]. A retrospective study comparing the three definitions of AKI found the largest proportion of cardiac surgery patients developed AKI using stage 1 KDIGO criteria [2]. Contrary to most of the studies on a heterogeneous population with variable baseline kidney function undergoing various cardiac surgery procedures, our study population comprised of those with elective isolated CABG only and all of them had a preserved baseline kidney function. For instance, among the studies included in the aforementioned meta-analysis, only $24 \%$ patients had undergone isolated CABG surgery [20]. The meta-analysis found similar incidences for AKI observation is consistent with our study where incidences of AKI were similar between KDIGO and AKIN.

Our descriptive findings showed that advanced age, comorbidities such as diabetes and hypertension, longer perfusion and cross-clamp time, use of intraoperative blood products, and prolonged ventilation were associated with higher incidence of AKI. Others have also identified similar associations [13, 20, 22]. Additional factors that have been associated with AKI include congestive heart failure and reduced left ventricular ejection fraction of less than $35 \%$, female gender, chronic obstructive pulmonary disease, prior cardiac surgery, and emergency nature of the procedure [22]. We, however, did not find such association in our study cohort since we only included patients having undergone elective isolated CABG procedures. Our study population had a male preponderance $(82.6 \%)$, a finding similar to those reported in large national registries like the Society of Thoracic Surgeons adult cardiac surgical database [23]. There is evidence to suggest that both referral and surgical revascularization rates of women with coronary artery disease are less than men [23-25].

We found that across all three definitions, AKI was significantly associated with higher mortality than those without AKI. This is in accordance with other published studies suggesting that the prognosis is much poorer for patients who develop AKI than those who do not $[13,16]$. We found that the discriminative power of the RIFLE criteria was superior to AKIN and KDIGO for 30-day mortality after surgery. However, that for overall morbidity it fell in undesirable ranges and was suggestive of low power of discrimination. Prior studies have yielded discrepant results when it comes to the prediction of outcomes in cardiac surgery patients with AKI. A study from Finland had reported that RIFLE classification discriminated 90-day mortality quite well (AUC 0.824 ) compared with the change of SCr and the change of eGFR AUCs ( 0.849 
and 0.829 , respectively) [1]. However, they did not compare the performance of RIFLE with AKIN and KDIGO [1]. Recently, a retrospective study on 1,551 cardiac surgery patients compared the incidence and effect on outcome (90-day and 1-year mortality) of different definitions. They found that RIFLE had the highest positive likelihood ratio of AKI to predict mortality [2]. They concluded that it was the best definition of AKI as determined by the ability to predict short-term mortality [2] However, they found KDIGO to be the most sensitive of the AKI definitions to identify the condition. However, the study population was heterogeneous having undergone diverse cardiac surgery procedures including valve replacements, heart transplants, etc., and only one-quarter of the subjects had had isolated CABG. Sampaio et al. [26] found KDIGO criteria to be superior to AKIN and RIFLE in a study based on 321 patients undergoing cardiac surgery, including valve replacement, myocardial revascularization, or both. Bastin et al. [27] reported a retrospective study on 1,881 adults who had cardiac surgery and observed no differences between KDIGO and AKIN criteria in this regard and both correlated better with mortality than did RIFLE; their study population was diverse and only less than half (47.6\%) had undergone isolated CABG. The 20th International Consensus Conference of the Acute Disease Quality Initiative Group recommended using KDIGO criteria to diagnose AKI in cardiac surgery patients since it demonstrates greater sensitivity to detect AKI and to predict associated in-hospital mortality than either the RIFLE or AKIN criteria [28]. Having said that, it is important to consider that most of the data, which made the basis for the recommendation, originated from North America, Europe, and upper-middle to upper income countries in Asia. There is paucity of data in this area from South Asia, which is home to a quarter of the world's population. Further research is needed to define the epidemiology of AKI after cardiac surgery in low-middle-income countries from Asia. Given that KDIGO classification did not alter the incidence or staging of AKI compared with AKIN criteria, we further propose that the role of the KDIGO classification for prediction of adverse outcomes in patients undergoing cardiac surgery needs to be explored further in future studies as well.

AKI requiring KRT although catastrophic in this context is relatively uncommon, occurring in 1-2\% of all patients undergoing surgery in most programs. Our findings were consistent with the reported evidence in this respect, as only 10 patients (1.1\%) required any form of RRT.
This is the first study from Pakistan and South Asia to compare the three criteria (AKIN, KDIGO, and RIFLE) regarding their ability to predict 30-day mortality and morbidity in cardiac surgery patients. Compared to other studies that had included a heterogeneous population undergoing a variety of cardiac surgical procedures, we only included isolated CABG patients.

To the best of our knowledge, this is the first study to compare three AKI definitions in CABG patients in same study. However, our findings should be interpreted with certain limitations: being a retrospective analysis, it has its own inherent limitations. Given that it was a singlecenter study, the results may not be generalizable. We reported crude estimates, which were not adjusted for comorbid conditions and therefore may have overestimated the magnitude of the AKI in the given population. Moreover, we did not use the urine output criteria for definitions of AKI. Most studies on AKI after cardiac surgery have excluded urine output criteria because use of mannitol in the priming fluid of the cardiopulmonary bypass may affect it making difficult to interpret.

\section{Conclusion}

We conclude that in a South Asian cohort of patients having undergone elective isolated CABG surgery, AKI was frequent and performance of AKIN and KDIGO criteria was comparable to diagnose AKI, while RIFLE definition, though overestimated the incidence of AKI, had excellent discriminatory power to predict mortality compared to other definitions. Moreover, RIFLE criteria based on change in eGFR helped identify a greater percentage of patients in early stages of AKI, and hence, it would allow for implementation of appropriate intervention and preventive measures that may translate into better patients' outcomes. Prospective multicenter studies on larger cohorts in the South Asian population aimed at identification of risk factors and validation of various AKI definitions in their ability to predict outcomes are warranted.

\section{Statement of Ethics}

This study was approved by the institutional review board of the ethical committee at Aga Khan University (approval number: 4732-Sur-ERC-17) and was conducted in accordance with the principles of the Declaration of Helsinki. The requirement of written informed consent was waived due to the retrospective nature of the study.
36

Cardiorenal Med 2022;12:29-37 DOI: $10.1159 / 000523828$
Yaqub/Hashmi/Kazmi/Aziz Ali/Dawood/ Sharif 


\section{Conflict of Interest Statement}

The authors declare that they have no competing interests.

\section{Funding Sources}

No funding was received for this study.

\section{Author Contributions}

S.Y.: Conception and design, interpretation, drafting, critical revision, and final approval. A.A.A.: Data interpretation, drafting, and final approval. S.H.: Data analysis and interpretation, drafting, and final approval. M.K.K.: Design, data acquisition, and final approval. T.D.: Design, data acquisition, and final approval. H.S.: Conception and design, final approval. All the authors read and approved the final manuscript.

\section{Data Availability Statement}

The datasets used and/or analyzed during the current study are available from the corresponding author on reasonable request.

\section{References}

1 Kuitunen A, Vento A, Suojaranta-Ylinen R Pettilä $V$. Acute renal failure after cardiac surgery: evaluation of the RIFLE classification. Ann Thorac Surg. 2006;81(2):542-6.

2 Sutherland L, Hittesdorf E, Yoh N, Lai T, Mechling A, Wagener G. Acute kidney injury after cardiac surgery: a comparison of different definitions. Nephrology. 2020;25(3):212-

3 Wu B, Chen J, Yang Y. Biomarkers of acute kidney injury after cardiac surgery: a narrative review. Biomed Res Int. 2019;2019: 7298635.

4 Jessani S, Levey AS, Bux R, Inker LA, Islam M, Chaturvedi N, et al. Estimation of GFR in South Asians: a study from the general population in Pakistan. Am J Kidney Dis. 2014; 63(1):49-58.

5 Bowdish ME, D'Agostino RS, Thourani VH, Schwann TA, Krohn C, Desai N, et al. STS adult cardiac surgery database: 2021 update on outcomes, quality, and research. Ann Thorac Surg. 2021;111(6):1770-80

6 Mandrekar JN. Receiver operating characteristic curve in diagnostic test assessment. J Thorac Oncol. 2010;5(9):1315-6.

7 Bellomo R, Ronco C, Kellum JA, Mehta RL, Palevsky P; Acute Dialysis Quality Initiative Workgroup. acute renal failure - definition, outcome measures, animal models, fluid therapy and information technology needs: the second international consensus conference of the acute dialysis quality initiative (ADQI) group. Crit Care. 2004;8(4):R204-12.

8 Mehta RL, Kellum JA, Shah SV, Molitoris BA, Ronco C, Warnock DG, et al. Acute kidney injury network: report of an initiative to improve outcomes in acute kidney injury. Crit Care. 2007;11(2):R31.

9 Molitoris BA, Levin A, Warnock DG, Joannidis M, Mehta RL, Kellum JA, et al. Improving outcomes from acute kidney injury. J Am Soc Nephrol. 2007;18(7):1992-4.

10 Valette X, du Cheyron D. A critical appraisal of the accuracy of the RIFLE and AKIN clas- sifications in defining "acute kidney insufficiency" in critically ill patients. J Crit Care. 2013;28(2):116-25.

11 Khwaja A. KDIGO clinical practice guidelines for acute kidney injury. Nephron Clin Pract. 2012;120(4):c179-84.

12 Van Biesen W, Vanholder R, Lameire N. Defining acute renal failure: RIFLE and beyond. Clin J Am Soc Nephrol. 2006;1(6):1314-9.

13 Amini S, Najafi MN, Karrari SP, Mashhadi ME, Mirzaei S, Tashnizi MA, et al. Risk factors and outcome of acute kidney injury after isolated CABG Surgery: a Prospective Cohort study. Braz J Cardiovasc Surg. 2019;34(1):70-5.

14 Hiew KC, Sachithanandan A, Arif M, Badmanaban B, Muiz A, Faisal I, et al. Acute kidney injury following coronary artery bypass graft surgery in a tertiary public hospital in Malaysia: an analysis of 1,228 consecutive cases. Med J Malaysia. 2016;71(3):126-30.

15 Ortega-Loubon C, Fernández-Molina M, Pañeda-Delgado L, Jorge-Monjas P, Carrascal Y. Predictors of postoperative acute kidney injury after coronary artery bypass graft surgery. Braz J Cardiovasc Surg. 2018;33(4):323-9.

16 Yue Z, Yan-Meng G, Ji-Zhuang L. Prediction model for acute kidney injury after coronary artery bypass grafting: a retrospective study. Int Urol Nephrol. 2019;51(9):1605-11.

17 Chew ST, Mar WM, Ti LK. Association of ethnicity and acute kidney injury after cardiac surgery in a South East Asian population. $\mathrm{Br}$ J Anaesth. 2013;110(3):397-401.

18 Anand SS, Yusuf S, Jacobs R, Davis AD, Yi Q, Gerstein $\mathrm{H}$, et al. Risk factors, atherosclerosis, and cardiovascular disease among Aboriginal people in Canada: the study of health assessment and risk evaluation in Aboriginal peoples (SHARE-AP). Lancet. 2001;358(9288): 1147-53.

19 Fernandes VR, Cheng S, Cheng YJ, Rosen B, Agarwal S, McClelland RL, et al. Racial and ethnic differences in subclinical myocardial function: the multi-ethnic study of atherosclerosis. Heart. 2011;97(5):405-10.
$20 \mathrm{Hu}$ J, Chen R, Liu S, Yu X, Zou J, Ding X. Global incidence and outcomes of adult patients with acute kidney injury after cardiac surgery: a systematic review and meta-Analysis. J Cardiothorac Vasc Anesth. 2016;30(1): 82-9.

21 Yuan SM. Acute kidney injury after pediatric cardiac surgery. Pediatr Neonatol. 2019; 60(1):3-11.

22 Thiele RH, Isbell JM, Rosner MH. AKI associated with cardiac surgery. Clin J Am Soc Nephrol. 2015;10(3):500-14.

23 ElBardissi AW, Aranki SF, Sheng S, O'Brien SM, Greenberg CC, Gammie JS. Trends in isolated coronary artery bypass grafting: an analysis of the society of thoracic surgeons adult cardiac surgery database. J Thorac Cardiovasc Surg. 2012;143(2):273-81.

24 Jabagi H, Tran DT, Hessian R, Glineur D, Rubens FD. Impact of gender on arterial revascularization strategies for coronary artery bypass grafting. Ann Thorac Surg. 2018;105(1):62-8.

25 Nicolini F, Vezzani A, Fortuna D, Contini GA, Pacini D, Gabbieri D, et al. Gender differences in outcomes following isolated coronary artery bypass grafting: long-term results. J Cardiothorac Surg. 2016;11(1):144.

26 Sampaio MC, Maximo CA, Montenegro CM, Mota DM, Fernandes TR, Bianco AC, et al. Comparison of diagnostic criteria for acute kidney injury in cardiac surgery. Arq Bras Cardiol. 2013;101(1):18-25.

27 Bastin AJ, Ostermann M, Slack AJ, Diller GP Finney SJ, Evans TW. Acute kidney injury after cardiac surgery according to risk/injury/ failure/loss/end-stage, acute kidney injury network, and kidney disease: improving global outcomes classifications. J Crit Care. 2013; 28(4):389-96.

28 Nadim MK, Forni LG, Bihorac A, Hobson C, Koyner JL, Shaw A, et al. Cardiac and vascular surgery-associated acute kidney injury: the 20th international consensus conference of the ADQI (Acute Disease Quality Initiative) group. J Am Heart Assoc. 2018;7(11):e008834. 